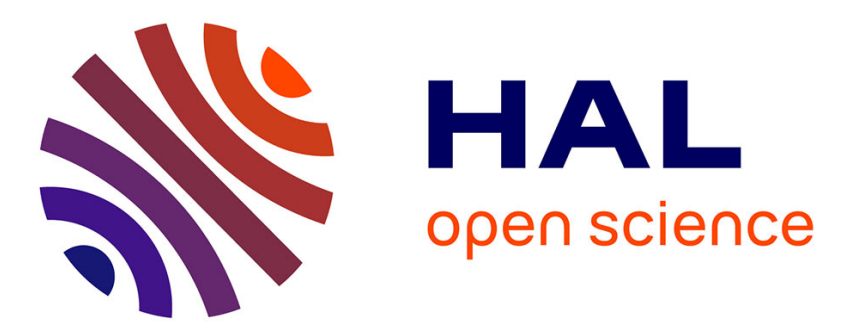

\title{
Geometrical deviation identification and prediction method for additive manufacturing
}

Zhicheng Huang, Jean-Yves Dantan, Alain Etienne, Mickaël Rivette, Nicolas

Bonnet

\section{- To cite this version:}

Zhicheng Huang, Jean-Yves Dantan, Alain Etienne, Mickaël Rivette, Nicolas Bonnet. Geometrical deviation identification and prediction method for additive manufacturing. Rapid Prototyping Journal, 2018, 24 (9), pp.1524-1538. 10.1108/RPJ-07-2017-0137 . hal-02303230

\section{HAL Id: hal-02303230 \\ https://hal.science/hal-02303230}

Submitted on 2 Oct 2019

HAL is a multi-disciplinary open access archive for the deposit and dissemination of scientific research documents, whether they are published or not. The documents may come from teaching and research institutions in France or abroad, or from public or private research centers.
L'archive ouverte pluridisciplinaire HAL, est destinée au dépôt et à la diffusion de documents scientifiques de niveau recherche, publiés ou non, émanant des établissements d'enseignement et de recherche français ou étrangers, des laboratoires publics ou privés. 


\title{
Geometrical deviation identification and prediction method for additive manufacturing
}

\author{
Zhicheng Huang \\ École Nationale Supérieure d'Arts et Métiers, Metz, France \\ Fean-Yves Dantan \\ Centre de Metz, Arts et Metiers ParisTech, Metz, France \\ Alain Etienne \\ Department of Computer Science, Arts et Metiers ParisTech, Metz, France, and \\ Mickaël Rivette and Nicolas Bonnet \\ Centre de Metz, Arts et Metiers ParisTech, Metz, France
}

\begin{abstract}
Purpose - One major problem preventing further application and benefits from additive manufacturing (AM) nowadays is that AM build parts always end up with poor geometrical quality. To help improving geometrical quality for AM, this study aims to propose geometrical deviation identification and prediction method for AM, which could be used for identifying the factors, forms and values of geometrical deviation of AM parts.

Design/methodology/approach - This paper applied the skin model-based modal decomposition approach to describe the geometrical deviations of $A M$ and decompose them into different defect modes. On that basis, the approach to propose and extend defect modes was developed. Identification and prediction of the geometrical deviations were then carried out with this method. Finally, a case study with cylinders manufactured by fused deposition modeling was introduced. Two coordinate measuring machine (CMM) machines with different measure methods were used to verify the effectiveness of the methods and modes proposed.

Findings - The case study results with two different CMM machines are very close, which shows that the method and modes proposed by this paper are very effective. Also, the results indicate that the main geometrical defects are caused by the shrinkage and machine inaccuracy-induced errors which have not been studied enough.

Originality/value - This work could be used for identifying and predicting the forms and values of AM geometrical deviation, which could help realize the improvement of AM part geometrical quality in design phase more purposefully.
\end{abstract}

Keywords Additive manufacturing, Geometrical defect modeling, Geometrical deviation prediction

\section{Introduction}

Additive manufacturing (AM) has already been studied and developed for almost 30 years and there is now a great amount of AM processes. The ability of manufacturing parts directly from computer aided design (CAD) models by commonly one single build step makes AM processes able to manufacture geometrically complex parts and this also makes AM more effective for single parts and small batches (Vayre et al., 2012; Gibson et al., 2010a). These advantages of AM pushed the global AM market grow 25.9 per cent to $\$ 5.165$ bn in 2015 (Wohlers, 2016), and AM processes have been applied in many different fields, such as automobile, aerospace, medical, academic and military (Guo and Leu, 2013).

Despite that AM has already been developed and applied extensively in many fields recent years, there are still some challenges for wider applications of AM processes, especially for building end-use parts (Gao et al., 2015). The varieties in manufacturing techniques, materials and process parameters, as well as build environment, lead to the poor repeatability and predictability with the same digital input (3D model) (Gibson et al., 2010b). Poor geometrical accuracy is also a major problem for AM considering that some factors would lead to geometrical defects of AM parts. Different factors such as "stair-stepping" effect (Boschetto et al., 2013), process variables and material properties (Gowda et al., 2014), support structure (Neri Volpato et al., 2014) as well as the surface approximation errors caused by CAD tessellation and slicing (Agrawal et al., 2014) would all influence the geometrical accuracy.

Plenty of research works have been carried out to study the dimensional and geometrical accuracy for AM build parts (Lieneke et al., 2015; Shahrain et al., 2016; Lieneke et al., 2016; Yang and Anam, 2014; Moylan et al., 2014). Most researchers

The authors would like to thank China Scholarship Council (CSC). This work is supported in part by the scholarship from CSC under the Grant CSC N²01406020103. 
developed various test artifacts to evaluate the AM part geometrical quality based on traditional geometrical dimensioning and tolerancing (GD\&T) characteristics (Yang and Anam, 2014).

Figure 1 (Lieneke et al., 2015) compares the geometrical quality that $\mathrm{AM}$ processes and different traditional manufacturing processes could meet. As shown in the table, the accuracy of AM processes is still limited, contrasting with traditional manufacturing processes. Developing methods for analyzing and estimating the geometrical deviations of additive manufactured parts is a possible way to improve geometrical quality for AM. This paper aims to introduce the $A M$ geometrical deviation identification and prediction method considering form defects. Geometrical deviation could be considered as the superposition of defects caused by different defect factors. Based on this, the method would analyze the factors that cause the geometrical deviations of AM parts and build defect modes for analyzing and estimating them considering the characteristics of AM process.

However, this study focuses not only on the estimation of AM parts geometrical quality considering traditional GD\&T characteristics but also on the identification of geometrical defect modes for realizing the geometrical quality optimization specifically in future work. Thus, a method to propose geometrical defect modes is necessary. In which, AM processes are first classified according to the build method, factors that would cause deviations should then be analyzed to help identify the deviation shapes. Geometrical defect modes caused by the process-oriented factors should be built to identify the form of the deviations. Geometrical deviation identification and prediction could then be carried out by fitting the prediction shape, generated by adding the defect modes to the nominal surface, with real shapes through iterative least square method.

This research proposes a geometrical defect mode build method to identify geometrical defect modes for AM considering characteristics of AM processes. Based on the modes proposed, method to identify and predict the AM manufactured part geometrical deviations is introduced. This paper mainly focuses on presenting the methodology to identify

Figure 1 IT classes for various manufacturing processes

\begin{tabular}{|c|c|c|c|c|c|c|c|c|c|c|}
\hline \multirow{2}{*}{ Processes } & \multicolumn{10}{|c|}{ IT class } \\
\hline & 7 & 8 & 9 & 10 & 11 & 12 & 13 & 14 & 15 & 16 \\
\hline \multicolumn{11}{|l|}{ Casting } \\
\hline \multicolumn{11}{|l|}{ Sintering } \\
\hline \multicolumn{11}{|l|}{ Drop forging } \\
\hline \multicolumn{11}{|l|}{ Milling } \\
\hline \multicolumn{11}{|l|}{ Cutting } \\
\hline \multicolumn{11}{|l|}{ Turning } \\
\hline \multicolumn{11}{|l|}{ Drilling } \\
\hline \multicolumn{11}{|l|}{ Planning } \\
\hline \multicolumn{11}{|l|}{ Stripping } \\
\hline \multicolumn{11}{|l|}{ AM processes } \\
\hline \multicolumn{11}{|l|}{ FDM } \\
\hline \multicolumn{11}{|l|}{ SLS } \\
\hline SLM & & & & & & & & & & \\
\hline
\end{tabular}

Source: Lieneke et al. (2015) and predict the geometrical deviations of AM process manufactured parts. This method could be used to define and contify the defect modes and modal representation, which were applied by Dantan et al. (2017) as the support of their modal representations. Their work validated the application of this study by using the case study resulting from this research and proposed a geometrical simulation tool to study the impacts of these deviations on the geometrical behavior of the AM products.

Being aware that material extrusion is the most popular AM process worldwide ever since early 2000s (Wohlers, 2016), this study takes fused deposition modeling (FDM) as an example to apply this method. Considering that cylinders are widely used in the products such as in the form of axis and applied in "pinhole" relationship to assembly different parts together, this paper would focus on the geometrical deviation of cylinder surface to illustrate the application of the methods.

The structure of this paper is as follow: Related researches about analyzing and estimating AM geometrical quality and works on deviation modeling are introduced in Section 2. The methodology of geometrical deviation identification and prediction method for AM is described is Section 3. Cylinder surface geometrical defect modes and the verification of proposed modes are detailed in Section 4. The results after implementing the proposed method and models with real parts are shown and analyzed in Section 5. Finally, the conclusion of this study is presented in Section 6.

\section{Literature review}

Geometrical deviations of AM are caused by different process factors. Geometrical quality analysis and estimation of AM, as well as the geometrical defect mode build method, are the bases to realize the identification and prediction of the geometrical deviation. There are already plenty of works proposed to study the geometrical quality of AM manufactured products and the geometrical deviation modeling.

\subsection{Additive manufacturing geometrical quality analysis and estimation}

Several AM geometrical quality analysis research works mainly focus on identifying process parameters that would significantly affect the geometrical quality, trying to estimate and improve the AM part quality with the process parameters proposed.

Mahmood et al. (Shahrain et al., 2016) proposed a hybrid experimental/theoretical approach to study the geometrical accuracy variation caused by process parameter change of FDM process built parts. This study shows that the top five common parameters component size, extruder temperature, print orientation and layer thickness would significantly affect the dimensional accuracy of printed parts.

Sahu et al. (2013) applied Taguchi method to study the influence of main process parameters of FDM such as layer thickness, orientation, raster angle, raster width and air gap on part dimensional accuracy. Prediction model based on fuzzy logic and Mamdani method was developed for improving dimensional accuracy. This method would need expert knowledge and experience to build massive rules for using fuzzy inference system, which might limit the application of this method. 
Campanelli et al. (2007) presented a statistical analysis of the stereolithographic process trying to find out the combination of process parameters for the best dimensional accuracy of the manufactured parts. Taguchi methodology was used for optimization procedure to get the specific values of process parameters which would help increasing the dimensional accuracy. And the results showed the availability to search for the best process parameters combination.

While these works could not identify the deviation forms, as well as the relationships between process characteristic and deviation shapes which are necessary for accurate analysis and estimation, other research works emphasized the form of geometrical deviation and proposed methods to analyze and estimate specific defects caused by particular AM process factors.

Xu et al. (2013) investigated shape deviation of parts built by Mask Image Projection Stereolithography (MIP-SLA), a variant of stereolithography. They considered over or under exposure, light blurring and phase-change-induced expansion or shrinkage as the main factors of shape deviation after studying the fabrication process. MIP-SLA-built cylinders and cubes were used for checking the deviation models, while this model-build method was not comprehensive enough to cover all defect forms with obvious effects.

Moroni et al. (2014) proposed a methodology to estimate dimensional and geometric deviation of AM part features by analyzing the STereoLithography (STL) input file. This work mainly considered the incorporating confounding errors caused by the "stair-step" effect and shrinkage of FDM process.

Except the deviations related to the AM process characteristic, AM machine errors would also directly affect the geometrical defect. Some works were carried out to specify the influence of AM machine inaccuracy (Tong et al., 2008; Song et al., 2014; Agrawal and Dhande, 2007, 2008). Tong et al. (2008) extended a slice file compensation method for STL file and SSL file to improve the accuracy of AM parts. In this work, the defects caused by AM machines are taken into consideration. All machine errors were mapped into a "virtual" parametric machine error model and a polynomial regression model. Two different AM machines, FDM 3000 machine and SLA 250 machine, were tested with the same input. This study shows that the machine control and movement resolution have a great impact on the part accuracy and even limit distinguishing the difference between STL file compensation and SSL file compensation.

Song et al. (2014) developed a systematic approach to model the dimensional errors for FDM. Two significant error sources that affect the shape of the product consecutively were identified after analyzing the mechanism of FDM machine: positioning error of the extruder and shape deformation caused by processing error. Kriging method was applied to predict the deviation of the extruder positioning error, whereas this method was based on the independent sparse points on the platform, and the defect shapes caused by machine errors could not be obtained directly.

Agrawal and Dhande (2007, 2008) introduced a unified stochastic approach to study the geometrical and mechanical error of AM process. The structure of AM machine was first studied to build the geometric model for the AM process description. Stochastic modeling of the process could be carried out using the central limit theorem. The closed-form expressions of the manufactured surface points traced by the laser beam or the tip of the nozzle head were then described with random variables involved in the process. This method could be used for specifying the distribution of AM process machine errors and can be extended for optimal allocation of tolerances and clearances.

These research works show that many efforts have been carried out to estimate and improve the geometrical deviations of endused products built by AM processes. There are many approaches that could be applied to analyze and estimate geometrical deviations for AM. However, few identified the different defect forms caused by model errors, AM processing induced errors and machine errors globally. To help estimating geometrical deviations of AM parts more accurately and optimizing process parameters more purposively, defect forms caused by different factors need to be specified and modeled properly.

\subsection{Geometrical deviation modeling}

Geometrical deviation of part is identified by the displacement between real surface and nominal surface. Thus, a real surface can be modeled by an ideal substituted surface with geometrical deviation which could be divided by a set of form modes. The description of geometrical form defects has long been studied for specifying tolerance.

Continuous substituted surface models does not allow taking into account form defects when modeling geometrical defects (Homri et al., 2016). Ballu and Mathieu (1993) proposed the skin model concept, which is developed from the theoretical foundations of geometrical product specification. This concept uses discrete geometry representation schemes for the representation of part geometry considering all different kinds of geometric deviations (Schleich et al., 2016) and could be used to specify geometrical defects with numerous mathematical descriptions. Qiao et al. (2016) developed a deviation coordinate system to represent the non-ideal cylindrical surface by adding a deviation dimension in the curvilinear coordinates instead of applying the Cartesian coordinate system. This description method is convenient for specific geometries such as cylinder and sphere, whereas the application to extend this method for describing complex shapes would be difficult.

Beside the system and concept for modeling the form defects, studies on the decomposition of form defects have also been carried out. Morière et al. (2010) described form defects with 2 degree polynomials which are applied to shift the nodes of a STL model. Huang and Ceglarek (2002) developed discrete-cosine-transformation-based decomposition method to modeling the part form defect. The geometrical defect is decomposed into a series of independent error modes, which allow a statistical distribution of the coefficients for each mode. This method was applied by Lecompte et al. (2010) for predicting plane surface form defect as a sum of individual technological defects. These defect decomposition methods could not describe and reflect the shape of deviations directly, making them inappropriate to be applied in the study.

Samper and Formosa (2007) and Formosa and Samper (2007) proposed the discrete modal decomposition, based on natural mode shapes of a discredited feature; this study also applied the finite elements method to compute the shapes and obtain the modal coefficients of the defects. Homri et al. (2017) 
developed metric modal decomposition (MMD) method to represent the defect shapes directly. Three types of modes are defined in the MMD method: rigid modes, modes of undulation and modes of section. This method has been applied to realize the simulation and optimization of a simplified over-constrained industrial mechanism assembly considering form defects.

These research works show that plenty of works have been carried out attempting to model and decompose the geometrical deviation of parts with different methods. Considering that the specification of defect shapes is necessary for accurately analyzing the defects caused by different factors, this study aims to adapt skin model concept proposed by Ballu and Mathieu (1993), as well as the MMD method proposed by Homri et al. (2017), which could help using abundant mathematical descriptions to build and reflect defect shapes directly.

\section{Geometrical deviation identification and prediction method for additive manufacturing}

This paper aims at proposing a method for building AM form defect modes considering different defect factors globally, as well as a geometrical deviation identification and prediction method to study the deviation caused by the defect modes. Geometrical deviation description method is presented in Section 3.1 which is the base to realize this study. The global method to identify and predict geometrical deviation with defect modes is introduced in Section 3.2. The details of applying the defect modes are introduced in Section 3.3.

\subsection{Geometrical deviation description method}

This study would discredit and represent geometrical shape with points on the surface. Specially, real surface of test parts is represented by the measurement result from coordinate measuring machines. Thus, the geometrical defects could be defined as the modal vector fields, which describe the displacement of the points mathematically with elementary defects modes. Take cylindrical surface as example: a substituted discrete surface is generated by the points on the nominal surface with different geometrical defect modes, as shown in Figure 2.

Mathematically:

$$
\mathbf{O} \mathbf{M}_{\mathbf{i}}^{\mathbf{R}}=\mathbf{O} \mathbf{M}_{\mathbf{i}}^{\mathbf{N}}+\sum_{k=0}^{n}\left(\lambda_{k, M_{i}}+\varepsilon_{k, M_{i}}\right) \cdot \mathbf{u}_{\mathbf{k}, \mathbf{M}_{\mathbf{i}}}
$$

Figure 2 Substituted surface description with geometrical deviation

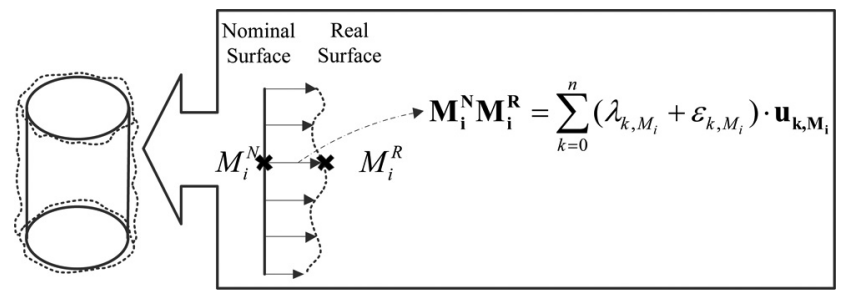

Source: Dantan et al. (2017)
In which:

$O \quad=$ original point of coordinate system;

$\mathbf{O} \mathbf{M}_{\mathbf{i}}^{\mathbf{N}}=$ coordinate of point $\boldsymbol{i}$ in the nominal surface;

$\mathbf{O M}_{\mathbf{i}}^{\mathbf{R}}=$ coordinate of point $\boldsymbol{i}$ in the real surface;

$\mathrm{u}_{\mathbf{k}, \mathbf{M}_{\mathbf{i}}}=$ geometrical deviation orientation by the $k^{\text {th }}$ geometrical defect mode applied on the point $\boldsymbol{i}$ of nominal shape;

$\lambda_{k, M_{i}}=$ systematic component amplitude of the $k^{\text {th }}$ defect mode applied on the point $\boldsymbol{i}$ of nominal shape; and

$\varepsilon_{k, M_{i}}=$ aleatory component amplitude of the $k^{\text {th }}$ defect mode applied on the point $i$ of nominal shape.

The geometrical deviation between substituted prediction surface and nominal surface is defined by the sum of deviations caused by all defect modes on each point. Based on this geometrical deviation description method, the geometrical deviation identification and prediction could be carried out.

\subsection{Geometrical deviation identification and prediction} To help in quantitatively studying the impact of the process parameters on the geometrical deviation of the AM parts, this study proposes geometrical defect modes according to the defect shapes caused by different process factors. Geometrical deviation identification and prediction method for $\mathrm{AM}$ is proposed, as shown in Figure 3; details of each step are introduced as below:

\subsubsection{Identify additive manufacturing process defect factors}

The process of manufacturing an end-used part with AM is shown in Figure 4; each manufacturing step would have different influence on the geometrical deviation of final parts. Based on the literature review (Campanelli et al., 2007), the AM geometrical

Figure 3 Geometrical deviation identification and prediction method for AM

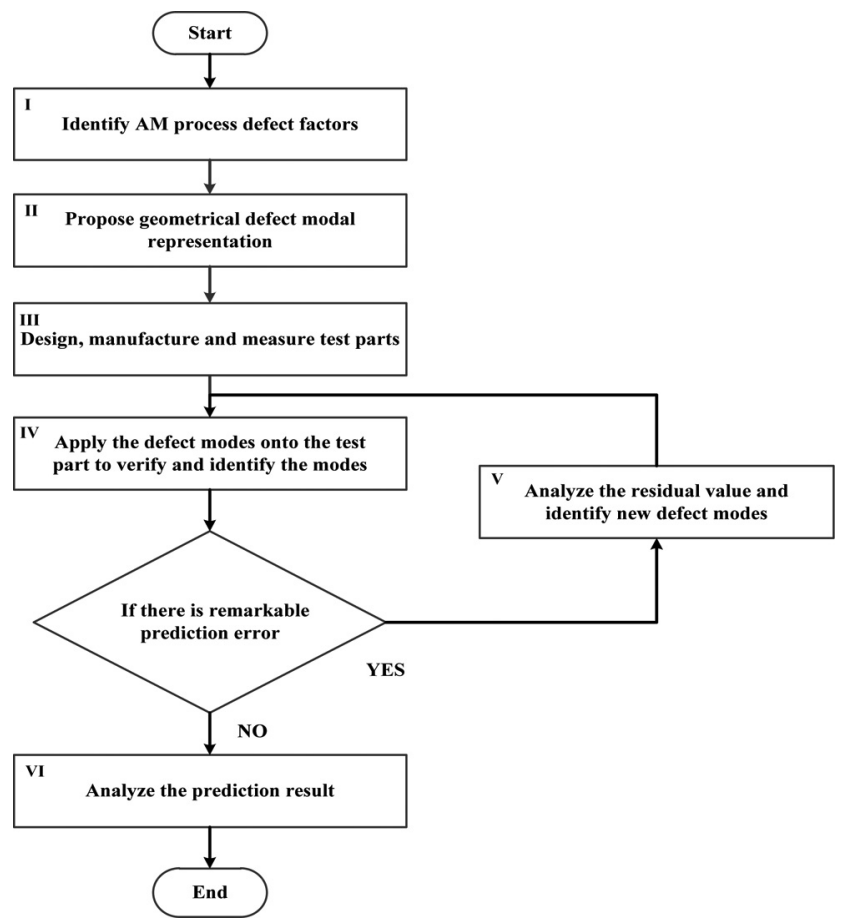


Figure 4 AM manufacturing process

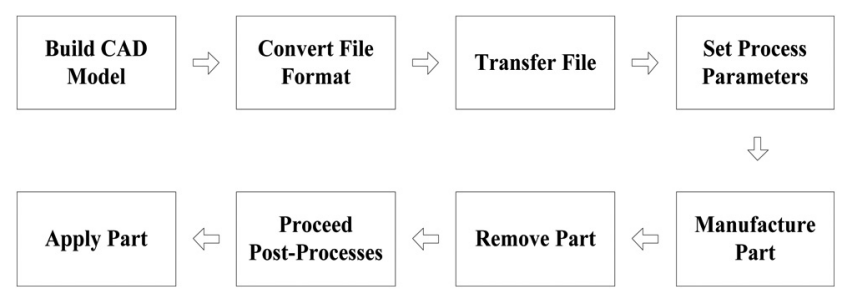

defect factors could be summarized as three parts: model error, AM processing induced errors and machine errors.

The model error is caused by the operations like CAD tessellation which would cause the surface approximation errors and lead to 3D file data deviation which are irrelevant with $A M$ process type.

The AM processing induced errors are strongly related to the process manufacture method. Thus, studying AM processes based on the build method would help reducing repetitive work for further study considering different AM processes with same build method would have similar geometrical defect forms. According to American Society for Testing and Materials (ASTM International) (ISO/ASTM52900-15, 2015), AM processes could be classified into seven types as the Table I shows by using the AM build technique as baseline.

The machine errors are mainly caused by the location and control problems, as well as the geometrical errors of machine, which are strongly related to the particular machine used. Conventional machine errors should be studied to propose proper defect forms. Also we should be aware of the specific error of a certain machine; method to identify ignored defect modes for completing the defect models is necessary which would be introduced in Step V.

\subsubsection{Propose and verify geometrical defect modal representations}

With the analysis above, major characteristics of geometrical defect forms could be summarized and proposed.

Take manufacturing cylinders with FDM as example, the model error is caused by the CAD tessellation, which uses polygons to approximate the cylinder sections.

Although machine errors are specific for particular machine, some common machine errors could be analyzed. For example, the serial FDM machine extruder movement involves two perpendicular directions, along $x$-axis and $y$-axis, respectively. The inaccurate control of movement would end up with the ellipse shape when the designed tool path is cycle. Specifically, geometry defects of machine itself would also increase the machine error.

While printing parts, the FDM uses material extrusion method, which uses build parts with heated molten materials deposited on a substrate. Based on the literature review (Xu et al., 2013), this processing method would mainly cause the "stair-stepping" effect and the part dimension change caused by the shrinkage while cooling down.

Based on the defect modes proposed, mathematical modal representation of the geometrical defect could be developed with the skin model concept. Before applying the defect modes on real parts, it is necessary to verify the accuracy of the defect mode representations. Details of the form defect modes, as well as the verification method, are introduced in Section 4.

\subsubsection{Design, manufacture and measure test parts}

To quantify and identify the geometrical defect modes properly, test parts should be designed to ensure both the conveniences of measurement and the conspicuousness of geometrical defect forms. Considering that cylinders could show defect shapes in different directions congruously, cylinders are applied to analyze this geometrical deviation identification and prediction method.

\subsubsection{Apply defect modes onto the test parts to identify the modes}

With the defect mode proposed, the prediction shape could be generated with all considered defect modes applied on the nominal shape, as shown in Figure 5.

Then the generated prediction shape would be fitted with the real surface to identify the geometrical deviations caused by different defect modes. Considering that iterative least square method could be used for providing the fitting shape solution with the least deviation from the target shape taking into account all the points at the same time successfully, this study would use this approach to verify and identify form defect modes proposed. Parameters, as well as the contribution of each mode, could be calculated properly for best fit. Details of this step are introduced in Section 3.3.

One should be aware that machine errors are strongly related to the particular machine used; specific machine errors might not be discovered first and cause notable residual value. After fitting the prediction shape and obtaining the distance between the prediction points and measured points, checking the existence of remarkable prediction error is necessary for completing the defect modes.

Table I Characteristics and main parameter of AM methods ("ISO/ASTM52900-15)

\begin{tabular}{|c|c|c|}
\hline Method & Build technique & Example \\
\hline Material extrusion & Continuous extrusion and deposition. Solidification by cooling & Fused deposition modeling (FDM) \\
\hline \multirow[t]{3}{*}{ Powder bed fusion } & Melting by beam & Selective laser melting (SLM) \\
\hline & Solidification by cooling & Selective laser sintering (SLS) \\
\hline & & Electron beam melting (EBM) \\
\hline Vat photopolymerization & Photopolymerization by laser & Stereolithography (SLA) \\
\hline Material jetting & Deposition solidification by cooling or photopolymerization & Material jetting (MJ) \\
\hline Binder jetting & Drop-on-demand glue binds powder & Binder jetting (BJ) \\
\hline \multirow[t]{2}{*}{ Sheet lamination } & Feeding and binding of sheets with adhesives & Ultrasonic additive manufacturing (UAM) \\
\hline & & Paper lamination technology (PLT) \\
\hline Directed energy deposition & Local powder injection and melted by laser & Direct metal deposition (DMD) \\
\hline
\end{tabular}


Figure 5 Prediction shape generation

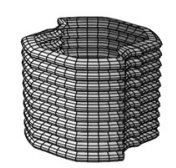

Prediction cylindrical surface

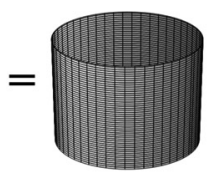

Nominal surface

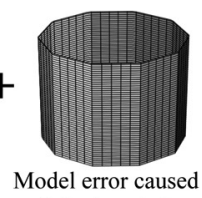

Model error caused defect mode 1

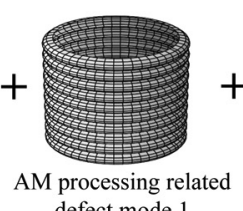
defect mode 1

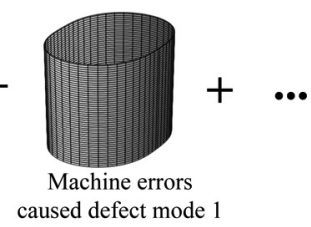

Source: Dantan et al. (2017)

Thus, if there is remarkable prediction error, the defect modes need to be extended and this method would start the loop and go to Step V to analyze the residual value. If there is no remarkable prediction error, the defect modes have already been proposed sufficiently, and this method would continue with Step VI.

\subsubsection{Analyze the residual value and propose new defect modes}

The existence of notable prediction error indicates the necessity to extend new modes. This step is then carried out to help identify the defect modes which are ignored. The residual values could be defined as the aleatory deviation plus the residual defect modes caused deviations. Mathematically:

$$
\mathbf{M}_{\mathbf{i}}^{\mathbf{R}} \mathbf{M}_{\mathbf{i}}^{\mathbf{P}}=\mathbf{\varepsilon}_{\mathbf{M}_{\mathbf{i}}}+\sum_{j=0}^{n} \lambda_{j, M_{i}}^{R} \cdot \mathbf{u}_{\mathbf{j}, \mathbf{M}_{\mathbf{i}}}^{\mathbf{R}}
$$

$$
\begin{aligned}
& \text { In which: } \\
& \mathbf{M}_{\mathbf{i}}^{\mathbf{N}} \mathbf{M}_{\mathbf{i}}^{\mathbf{P}}=\text { residual value on the point } i \text { of nominal surface }
\end{aligned}
$$

Based on the previous study (Tong et al., 2008), the main geometrical defects that need to be extended would be the machine errors caused defects, as well as the AM processinduced defects when the model error has been well applied. Compared with the irregular aleatory deviation appearance, defects caused by these factors should be periodic and remarkable considering the regularity and the repeatability of machine movement while processing. The frequency of large distance in residual value indicates the factors of new defect mode. Thus it is necessary to study the frequency of notable defects to help identify the new defect modes.

Considering that Fast Fourier transform (FFT) algorithm could be expediently applied to quantitatively analyze the frequency, this work would use it to study the notable frequency in the residual value.

To apply FFT algorithm, the angle angel between $\overrightarrow{O M_{i}^{N}}$ and $x$-axis is defined as $\theta_{M_{i}^{N}}$, the residual value $\mathbf{M}_{\mathbf{i}}^{\mathbf{R}} \mathbf{M}_{\mathbf{i}}^{\mathbf{P}}$ is defined as energy on the angle $\theta_{M_{i}^{N}}$ and the FFT algorithm is applied to study the frequency of high energy appearance. For example, Figure 6 is a result of residual value analysis with FFT algorithm, which shows high energy in the frequency of four times one cycle.

This FFT algorithm would be applied only when there is notable prediction error which indicates the necessity to build a new defect mode. With the frequency and shape of large deviations between real points and prediction points, new form defect models ignored previously could be highlighted. Machine knowledge and the specific AM machine processing movement, as well as the AM process characteristics, need to be studied for identifying proper defect factors. New defect modes could then be built on the basis of the residual analysis and the new found defect factors.

Take Figure 6 as example, the frequency indicates that the residual defect mode would cause large deviation four times as a cycle. After analyzing the machine movement, it is figured out that the untimely movement control, while the nozzle moves along $\mathrm{x}$ - and $\mathrm{y}$-axes would cause the rounded rectangle mode, introduced in Section 4.1.5, was not identified and resulted in this residual value frequency.

After the new defect modes proposed, this method would go back to Step IV to verify all the modes, and this loop would end only when there is no notable prediction error after the identification in Step IV.

\subsubsection{Analyze the prediction result}

With sufficient defect modes, this method could realize prediction and identification of geometrical defects caused by different factors. Parameters, as well as the contribution of each defect mode applied on AM parts, could be obtained. It is possible to identify main defect factors and carry out geometrical quality improvement work more effectively in future work with the method. Relationship between process parameters and geometrical deviations could be built more appropriately based on the prediction and identification result.

\subsection{Geometrical defect mode application method}

The process to quantify the geometrical defect modes is shown in Figure 7, and steps to realize the identification and prediction are introduced as below:

\subsubsection{Partition points}

Real surfaces are represented by the point cloud measured by coordinate measuring machines. This study mainly focuses on the geometrical deviation of AM cylindrical surfaces; point cloud result from the measurement could not be directly used considering the existence of unnecessary points. Point partition operation needs to be carried out, as show in Figure 8. Plenty of open source tools are available for the point cloud partition operation, such as MeshLab, CloudCompare and Slic3r. New 
Figure 6 Fast Fourier transform analysis result example

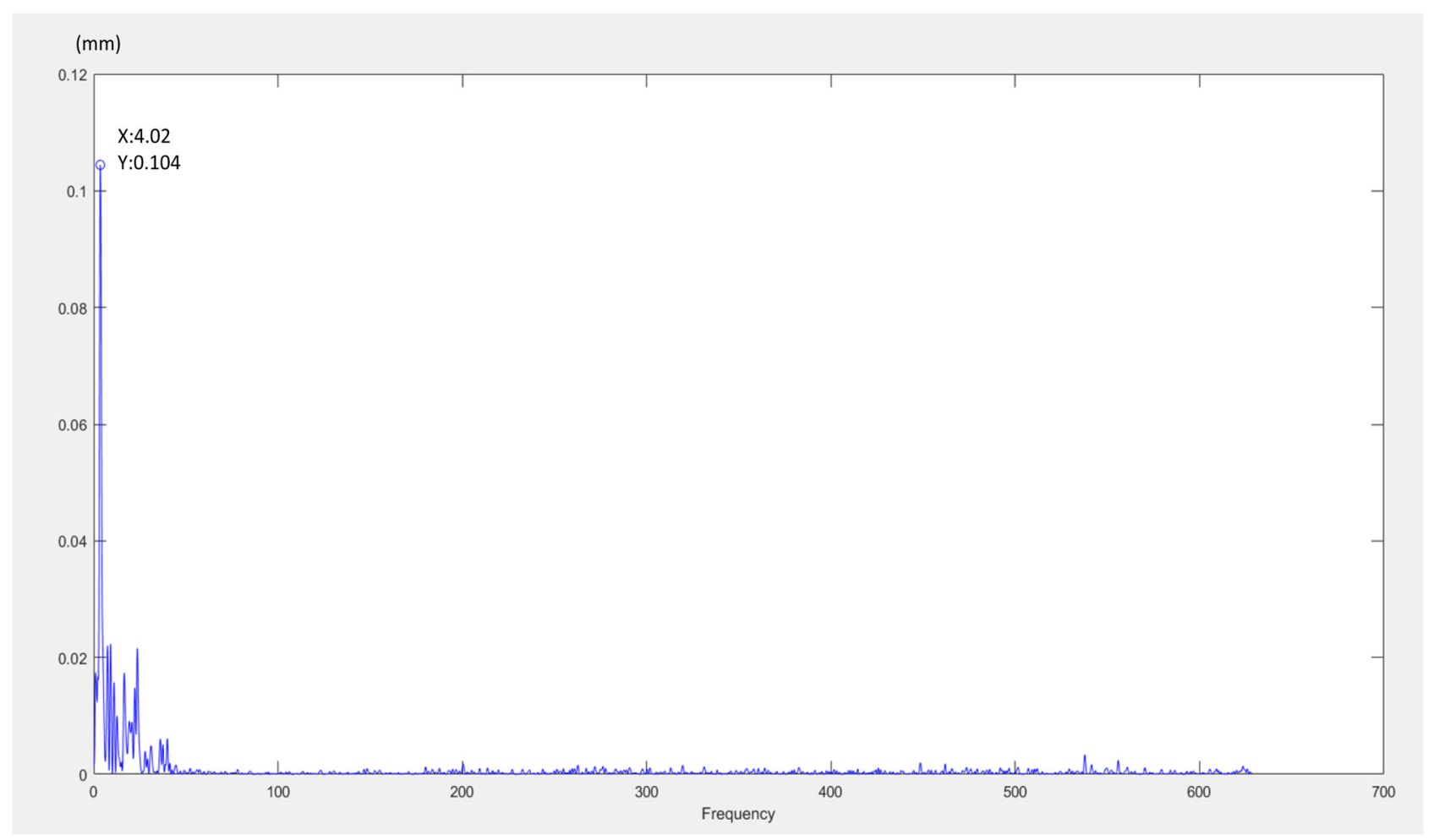

Figure 7 Modes variation and identification process

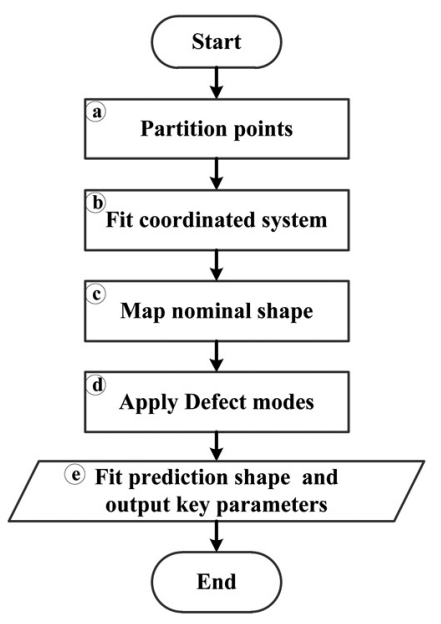

point clouds with only cylindrical surface information would be generated as the input for next steps.

\subsubsection{Fit coordinated system}

Specially, this study would like to fit the cylinder axis onto the $z$-axis and each cylinder bottom onto the XY plane for calculation convenience. Least square method is applied to identify the coordinate of cylinder axis. The objective to minimize is the quadratic sum of the distance between the measured cylindrical points and the fitting cylinder. Mathematically, the fitting cylindrical surface axis could be described with the intersection point on XY plane $\left(x_{o}, y_{o}, 0\right)$ and
Figure 8 Points partition

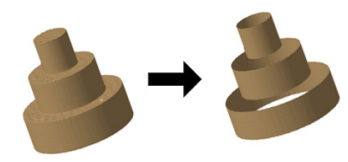

a vector $(i, j, 1)$. Define the fitting cylinder radius as $r_{o}$, the formula to be minimized is then as below:

$\sum\left\{\sqrt{\frac{\left[\left(X_{M_{i}^{C}}-x_{o}\right) * j-\left(Y_{M_{i}^{c}}-y_{o}\right) * i\right]^{2}+\left(Y_{M_{i}^{c}}-y_{o}-Z_{M_{i}^{c}} * j\right)^{2}+\left[\left(Z_{M_{i}^{c}} * i-\left(X_{M_{i}^{c}}-x_{o}\right)\right]^{2}\right.}{i^{2}+j^{2}+1}}-r_{o}\right\}$

In which, $X_{M_{i}^{C}}, Y_{M_{i}^{C}}, Z_{M_{i}^{C}}$ are the coordinate value of the cylindrical measured point $M_{i}^{C}$.

After obtaining the fitting cylinder axis coordinate value, rotation and movement are carried out to make the cylinder axis coincide with $z$-axis and the cylinder bottom in the XY plane, as shown in Figure 9.

The measured points after this operation could then be defined as:

Figure 9 Coordinated system fitting

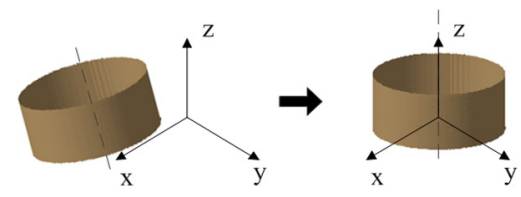




$$
\begin{aligned}
& {\left[\begin{array}{c}
X_{M_{i}^{R}} \\
Y_{M_{i}^{R}} \\
Z_{M_{i}^{R}}
\end{array}\right]^{T}=\left(\left[\begin{array}{c}
X_{M_{i}^{C}} \\
Y_{M_{i}^{C}} \\
Z_{M_{i}^{C}}^{T}
\end{array}\right]^{T}-\left[\begin{array}{c}
x_{o} \\
y_{o} \\
0
\end{array}\right]^{T}\right)\left[\begin{array}{ccc}
1 & 0 & 0 \\
0 & \frac{1}{\sqrt{j^{2}+1}} & \frac{j}{\sqrt{j^{2}+1}} \\
0 & -\frac{j}{\sqrt{j^{2}+1}} & \frac{1}{\sqrt{j^{2}+1}}
\end{array}\right]\left[\begin{array}{ccc}
\frac{\sqrt{j^{2}+1}}{\sqrt{i^{2}+j^{2}+1}} & 0 & \frac{i}{\sqrt{i^{2}+j^{2}+1}} \\
0 & 1 & 0 \\
-\frac{i}{\sqrt{i^{2}+j^{2}+1}} & 0 & \frac{\sqrt{j^{2}+1}}{\sqrt{i^{2}+j^{2}+1}}
\end{array}\right]} \\
& {\left[\begin{array}{c}
X_{M_{i}^{R}} \\
Y_{M_{i}^{R}} \\
Z_{M_{i}^{R}}
\end{array}\right]^{T}=\left[\begin{array}{c}
X_{M_{i}^{R}} \\
Y_{M_{i}^{R}} \\
Z_{M_{i}^{R}}^{T}
\end{array}\right]^{T}-\left[\begin{array}{c}
0 \\
0 \\
\min \left(Z_{M_{i}^{R}}\right)
\end{array}\right]^{T}}
\end{aligned}
$$

In which: $X_{M_{i}^{R}}, Y_{M_{i}^{R}}, Z_{M_{i}^{R}}$ are the coordinate value of the fitted measured point $M_{i}^{R}$ in the fitting system, $\left(x_{o}, y_{o}, 0\right)$ is the intersection point of the axis on XY plane and $(i, j, 1)$ is the vector of the axis.

\subsubsection{Map nominal shape}

Because the modal representation method needs to add defects on nominal points, it is necessary to locate the corresponding nominal points of measured points. One possible way is to map the measured points onto the nominal cylinder directly. With this method, the direction of defect vectors should be uniformed with the direction between measured points and corresponding nominal points. Thus, the corresponding point $M_{i}^{N}$ in the nominal substituted surface of the point $M_{i}^{R}$ in the real surface could be defined as the point mapped onto the nominal cycle in the height of $M_{i}^{R}$, parallel to the XY plane, as shown in Figure 10.

Mathematically:

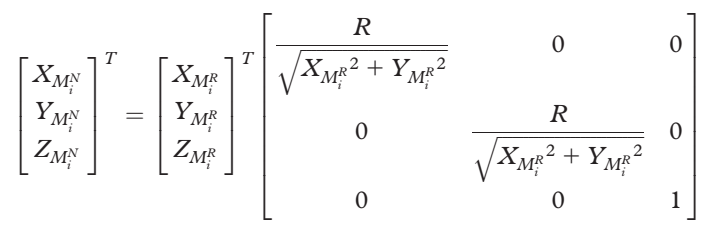

\subsubsection{Apply defect modes}

With the definition of real surface points and corresponding nominal substituted surface points, the prediction of geometrical deviation could be defined by the defects caused by all proposed geometrical defect modes applied on the discrete nominal surface:

$$
\mathbf{M}_{\mathbf{i}}^{\mathbf{N}} \mathbf{M}_{\mathbf{i}}^{\mathbf{P}}=\sum_{k=0}^{n} \lambda_{k, M_{i}} \cdot \mathbf{u}_{\mathbf{k}, \mathbf{M}_{\mathbf{i}}}
$$

In which:

$$
\begin{aligned}
\mathbf{M}_{\mathbf{i}}^{\mathbf{N}} \mathbf{M}_{\mathbf{i}}^{\mathbf{P}}= & \text { prediction of the geometrical deviation on the } \\
& \text { point } i \text { of nominal surface; }
\end{aligned}
$$

\section{Figure 10 Nominal shape mapping}

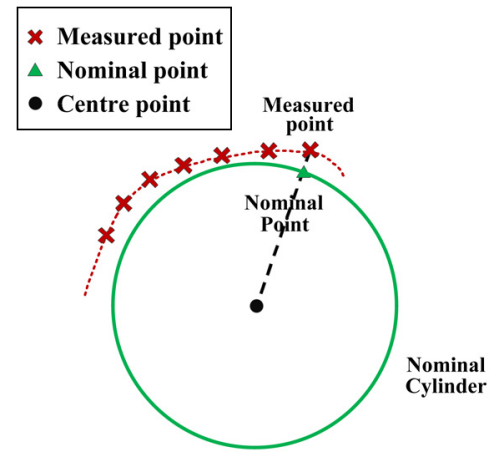

$\mathrm{u}_{\mathbf{k}, \mathbf{M}_{\mathbf{i}}}=$ geometrical deviation orientation by the $k^{\text {th }}$ geometrical defect mode applied on the point $i$ of nominal surface; and

$\lambda_{k, M_{i}}=$ amplitude of the $k^{\text {th }}$ defect mode applied on the point $i$ of nominal shape.

\subsubsection{Fit prediction shape and output key parameters}

Based on the concept of substituted discrete surface, a discrete prediction surface could be defined as the nominal substituted surface with geometrical deviation caused by defect modes proposed, as shown in Figure 11.

Mathematically, point on the prediction surface is defined as:

$$
\mathbf{O} \mathbf{M}_{\mathbf{i}}^{\mathbf{P}}=\mathbf{O} \mathbf{M}_{\mathbf{i}}^{\mathbf{N}}+\mathbf{M}_{\mathbf{i}}^{\mathbf{N}} \mathbf{M}_{\mathbf{i}}^{\mathbf{P}}=\mathbf{O} \mathbf{M}_{\mathbf{i}}^{\mathbf{N}}+\sum_{k=0}^{n} \lambda_{k, M_{i}} \cdot \mathbf{u}_{\mathbf{k}, \mathbf{M}_{\mathbf{i}}}
$$

In which:

$O \quad=$ original point of coordinate system;

$\mathbf{O M} \mathbf{P}=$ coordinate of point $i$ in the prediction surface;

$\mathbf{O} \mathbf{M}_{\mathbf{i}}^{\mathbf{N}}=$ coordinate of point $i$ in the nominal surface;

$\mathrm{u}_{\mathbf{k}, \mathbf{M}_{\mathbf{i}}}=$ geometrical deviation orientation by the $k^{\text {th }}$ geometrical defect mode applied on the point $i$ of nominal surface;

$\lambda_{k, M_{i}}=$ amplitude of the $k^{\text {th }}$ defect mode applied on the point $i$ of nominal shape; and

$\boldsymbol{\varepsilon}_{\mathbf{M}_{\mathbf{i}}} \quad=$ residual value of point $i$ after applying defect modes.

As introduced in Section 3.2, this study uses iterative least square method to fit the prediction points to the measured points, and the value to be minimized while applying the defect modes should be the quadratic sum of distance between the real surface points and the prediction points, which is:

$$
\sum_{i=0}^{N}\left\|\mathbf{M}_{\mathbf{i}}^{\mathbf{R}} \mathbf{M}_{\mathbf{i}}^{\mathbf{P}}\right\|
$$

Deviation caused by different defect modes, as well as the value of defect mode parameters, could be obtained successfully with this step.

This proposed method could be used to build geometrical defect modes considering different defect factors, and carry out the identification and prediction of AM part geometrical deviations. Proper geometrical defect modes are the basic to realize the prediction and identification of geometrical deviation caused by different factors.

Figure 11 Prediction surface generation

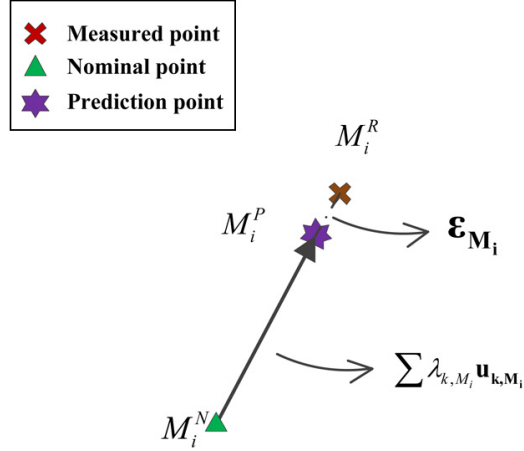




\section{Form defect modes description and verification}

To study the defects caused by different factors purposeful, form defect modes which would directly reflect the nature shape of defects caused by model error, AM process induced errors and machine errors should be built, respectively. Also, the feasibility as well as the accuracy of the proposed modes applied in the geometrical deviation identification and prediction method should be verified before applied with real AM parts.

\subsection{Form defect modes description}

As introduced in Section 3, this study locates corresponding nominal points by directly mapping the measured points on the nominal surface. Thus, the direction of the defect vectors should be defined uniformly with the direction between measured points and corresponding nominal points. One should be aware that this study would fit the cylinder axis to $z$-axis, and the defect orientation should also be uniform with the direction between nominal points and $z$-axis.

In the mathematic formulas introduced below, $R$ is the $\underset{\text { radius }}{S}$ of nominal cylindrical surface, $\theta_{M_{i}^{N}}$ is the angel between $O M_{i}^{N}$ and $x$-axis and $X_{M_{i}^{N}}, Y_{M_{i}^{N}}, Z_{M_{i}^{N}}$ are the coordinate values of the nominal point $M_{i}^{N}$.

The symbol per cent used in this paper is for modulo operation.

\subsubsection{Meshing mode}

This kind of geometrical defect is caused by the CAD system tessellation while transforming CAD models into STL files which is the format read by AM machines, as shown in Figure 12. Thus, the section of cylinders would turn into polygons. The key parameter for this defect mode is the number of polygon edges $n_{e}$ which is decided by the part dimension and the tessellation accuracy.

The geometrical defect of the meshing mode applied on nominal point $M_{i}^{N}$ could be mathematically described as:

$$
\begin{aligned}
\lambda_{1, M_{i}}= & \frac{R \cdot \sin \left(\frac{n_{e} \cdot \pi-2 \pi}{2 n_{e}}\right)-R \cdot \cos \left(\theta_{M_{i}^{N}} \%\left(\frac{2 \pi}{n_{e}}\right)\right)}{\cos \left(\theta_{M_{i}^{N}} \%\left(\frac{2 \pi}{n_{e}}\right)\right)} \\
\mathbf{u}_{\mathbf{1}, \mathbf{M}_{\mathbf{i}}}= & {\left[\begin{array}{c}
\frac{X_{M_{i}^{N}}}{R} \\
\frac{Y_{M_{i}^{N}}}{R} \\
0
\end{array}\right]^{T} }
\end{aligned}
$$

Figure 12 Meshing mode form

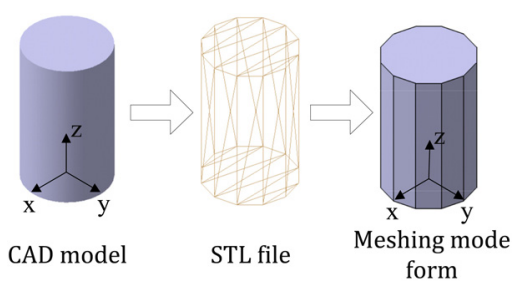

\subsubsection{Layer mode}

Manufacturing parts with AM processes "Stair-Stepping" effect caused by the "layer upon layer" build method is a defect that could not be ignored even with proper build direction, especially for material extrusion processes whose accuracy is strongly related to the material properties. The defect form of "Stair-Stepping" effect while manufacturing cylinders along $z$ axis with FDM is as shown in Figure 13. Considering that while extruded from nozzle, the heated material is not rigid, factors such as gravity would turn the material section into ellipse. Thus, the main parameters of this mode are the layer thickness $l$ and the XY plane paralleled semi-axis $d$ of material section.

Mathematically, the layer mode caused geometrical defect on the nominal point $M_{i}^{N}$ could be described as:

$$
\begin{aligned}
\lambda_{2, M_{i}}= & d \cdot \sqrt{1-4 \times\left(\frac{Z_{M_{i}^{N}} \% l}{l}\right)^{2}} \\
\mathrm{u}_{2, \mathbf{M}_{\mathbf{i}}}= & {\left[\begin{array}{c}
\frac{X_{M_{i}^{N}}}{R} \\
\frac{Y_{M_{i}^{N}}}{R} \\
0
\end{array}\right] }
\end{aligned}
$$

\subsubsection{Radius change mode}

Considering that plenty of AM process would heat material before building and the solidification takes place by cooling down, defect caused by the shrinkage is a main geometrical defect factor for AM. The defect form of shrinkage should be dimensional change, whereas the material used for manufacturing AM part is uniform. Thus, the form of this shrinkage caused mode is radius change in this study, as shown in Figure 14, and the key parameter for this radius change mode is the new radius $R^{\prime}$.

This radius change mode caused defect on the nominal point $M_{i}^{N}$ could be described as below:

$$
\begin{aligned}
\lambda_{3, M_{i}}= & R^{\prime}-R \\
\mathbf{u}_{3, \mathbf{M}_{\mathbf{i}}}= & {\left[\begin{array}{c}
\frac{X_{M_{i}^{N}}}{R} \\
\frac{Y_{M_{i}^{N}}}{R} \\
0
\end{array}\right] }
\end{aligned}
$$

Figure 13 Layer mode form
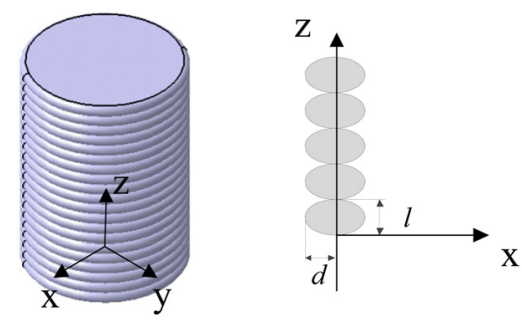
Figure 14 Radius change mode form
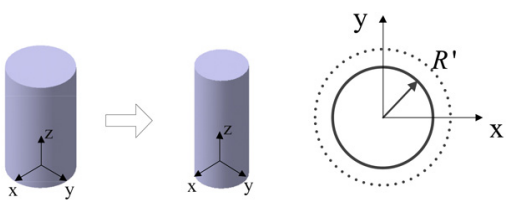

\subsubsection{Ellipse mode}

Except the AM manufacture characteristics caused geometrical defect modes introduced above, deviations caused by machine errors could also not be ignored because the AM machine movement controls the geometrical shape of parts directly. For some AM machines with low accuracy, designed cylinder tool path would always result in ellipse section, as shown in Figure 15, because of the inconsistent travel along $\mathrm{X}$ and $\mathrm{Y}$ directions while manufacturing.

The main parameter is the ellipse semi-axis along $y$-axis $R_{s}$, whereas assuming the semi-axis along $\mathrm{X}$ axis is still $R$. Then the defect caused by ellipse mode on the nominal point $M_{i}^{N}$ is introduced below:

$$
\begin{aligned}
\lambda_{5, M_{i}}=\left\{\begin{array}{l}
\frac{R}{\cos \left(\theta_{M_{i}^{\mathrm{N}}} \% \frac{\pi}{2}\right)}-R \\
\sqrt{2} \times\left(R-R_{r}\right) \cdot \cos \left(\theta_{M_{i}^{\mathrm{N}}} \% \frac{\pi}{2}-\frac{\pi}{4}\right) \\
+\sqrt{R_{r}^{2}-2 \times\left(R-R_{r}\right)^{2} \cdot \sin ^{2}\left(\theta_{M_{i}^{N}} \% \frac{\pi}{2}-\frac{\pi}{4}\right)}-R
\end{array}\right. \\
=\left[\begin{array}{c}
\frac{X_{M_{i}^{N}}}{R} \\
\frac{Y_{M_{i}^{N}}}{R} \\
0
\end{array}\right]
\end{aligned}
$$$$
\text { with } \theta_{M_{i}^{N}} \% \frac{\pi}{2} \in\left[0, \arctan \frac{R-R_{r}}{R}\right] \cup\left[\frac{\pi}{2}-\arctan \frac{R-R_{r}}{R}\right)
$$$$
\text { with } \theta_{M_{i}^{\mathrm{N}}} \% \frac{\pi}{2} \in\left(\arctan \frac{R-R_{r}}{R}, \frac{\pi}{2}-\arctan \frac{R-R_{r}}{R}\right)
$$$$
\begin{aligned}
\lambda_{4, M_{i}}= & R \cdot R_{s} \cdot \sqrt{\frac{1}{R_{s}^{2} \cdot \cos ^{2} \theta_{M_{i}^{N}}+R^{2} \cdot \sin ^{2} \theta_{M_{i}^{N}}}}-R \\
\mathrm{u}_{5, M_{\mathbf{i}}}= & {\left[\begin{array}{c}
\frac{X_{M_{i}^{N}}}{R} \\
\frac{Y_{M_{i}^{N}}}{R} \\
0
\end{array}\right]^{T} }
\end{aligned}
$$

\subsubsection{Rounded rectangle mode}

Except the ellipse mode, the rounded rectangle form defect mode as shown in Figure 16 also exists in some AM machines. This defect mode is due to the untimely movement control while the nozzle moves along $x$ - and $y$-axes. The key parameter defined in this mode is the fillet radius of the rounded rectangle section $R_{r}$. Thus, the geometrical defect by rounded rectangle mode applied on the nominal point $M_{i}^{N}$ could be proposed as below:

\subsubsection{Axis gap mode}

Geometrical defects of AM machine itself also have notable influence on the AM parts. For example, for the orthogonal structure FDM machines, as shown in Figure 17, one major geometrical defect of the parts manufactured by this type of machines is the deviation caused by the gap in the moving axis screw.

Figure 15 Ellipse mode form
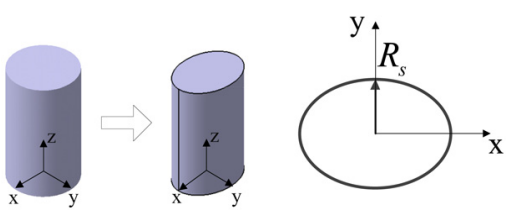

Figure 16 Rounded rectangle mode form

While the nozzle moves to a certain position it would jump because of the gap, the part would then form with the axis gap mode, as shown in Figure 18.

The main parameters for this mode are the gap distance $d_{g}$ and the gap angle $\alpha$. Mathematically, the axis gap mode caused geometrical defect on the nominal point $M_{i}^{N}$ could be described as below.

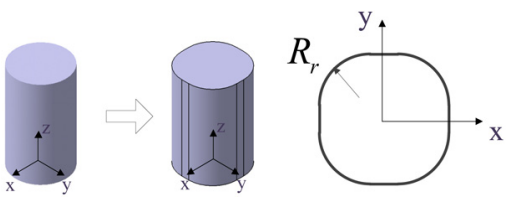




$$
\begin{aligned}
& \int \frac{d_{g}}{2} \cdot \sin \theta_{M_{i}^{N}}+\sqrt{R^{2}-\frac{d_{g}^{2}}{4} \cos ^{2} \theta_{M_{i}^{N}}}-R \quad \text { with } \theta_{M_{i}^{N}} \in\left[0, \frac{\pi}{2}-\frac{\alpha}{2}\right) \cup\left(\frac{3 \pi}{2}+\frac{\alpha}{2}, 2 \pi\right) \\
& \frac{l_{1} \cdot l_{2} \cdot \sin \alpha}{l_{1} \cdot \sin \left(\frac{\pi}{2}+\frac{\alpha}{2}-\theta_{M_{i}^{N}}\right)+l_{2} \cdot \sin \left(\theta_{M_{i}^{N}}+\frac{\alpha}{2}-\frac{\pi}{2}\right)}-R \quad \text { with } \theta_{M_{i}^{N}} \in\left[\frac{\pi}{2}-\frac{\alpha}{2}, \frac{\pi}{2}+\frac{\alpha}{2}\right]
\end{aligned}
$$

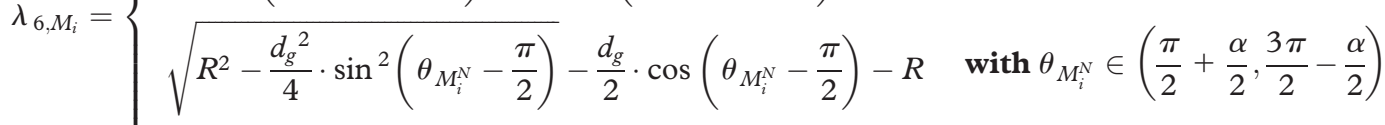

$$
\begin{aligned}
& \frac{l_{1} \cdot l_{2} \cdot \sin \alpha}{l_{1} \cdot \sin \left(\frac{3 \pi}{2}+\frac{\alpha}{2}-\theta_{M_{i}^{N}}\right)+l_{2} \cdot \sin \left(\theta_{M_{i}^{N}}+\frac{\alpha}{2}-\frac{3 \pi}{2}\right)}-R \quad \text { with } \theta_{M_{i}^{N}} \in\left[\frac{3 \pi}{2}-\frac{\alpha}{2}, \frac{3 \pi}{2}+\frac{\alpha}{2}\right]
\end{aligned}
$$

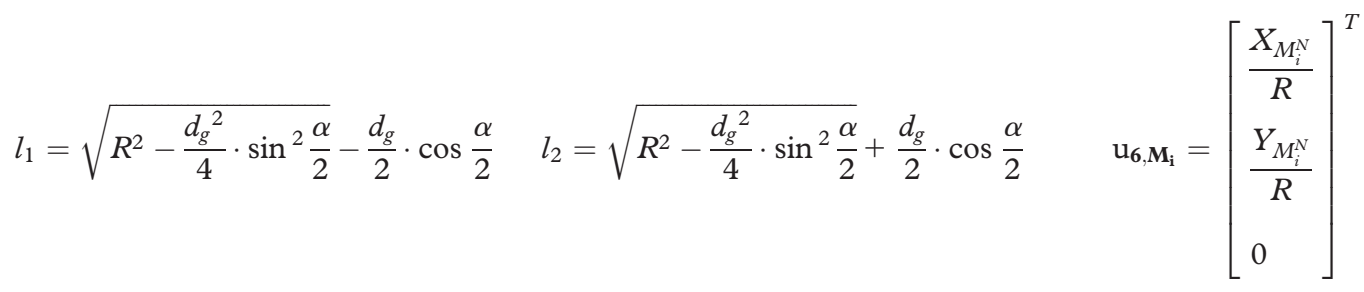

These defect modes are proposed using the geometrical deviation identification and prediction method introduced in Section 3. Considering that AM processing is automatic, the only way to improve the geometrical quality of AM parts is to modify the process parameters and the CAD model in design phase. While some defect mode parameters could not straightly reflect the AM process parameters and input model parameters, the relationships between process parameters and the defect modes need to be studied to help directly improving the geometrical quality of AM parts in future work. Based on the AM process knowledge, the possible process parameters that would influence each defect mode could be concluded, as shown in Figure 19, we have already finished the design of experiments and now identifying the relationships between the process parameters and the defect modes.

Figure 17 A schematic of the serial FDM machine

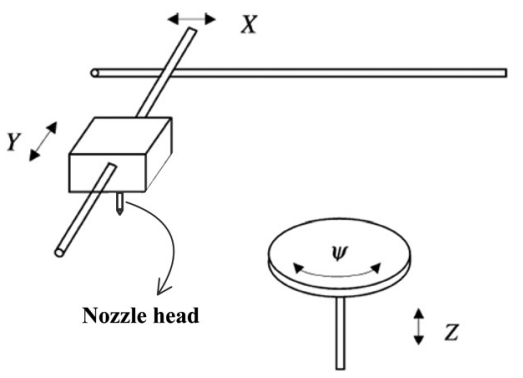

Figure 18 Axis gap mode form
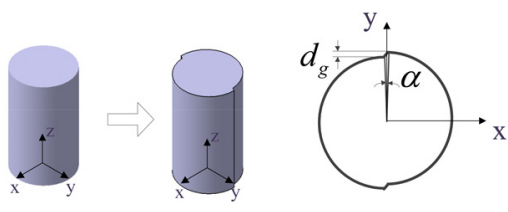

\subsection{Defect mode verification}

To ensure the feasibility of the geometrical deviation prediction and identification method, the defect mode descriptions proposed need to be applied and verified before real case application. This verification first applies the geometrical defects on nominal shapes in CAD system and generates defected STL files as the "measured point clouds," as shown in Figure 20.

Then Step IV “Apply the defect modes onto the test part to identify the modes" in Section 3 would be carried out to generate identified defect mode parameter values, as shown in Figure 21.

Values resulting from the "Apply the defect modes onto the test part to identify the modes" step would be compared with the defect mode values applied on the STL files to check the feasibility and the accuracy of the defect modes proposed. Table II shows the comparison of the designed defect parameter values and the identification result of each defect mode, which uses radius $40 \mathrm{~mm}$ and height $120 \mathrm{~mm}$ as nominal cylinder shape. The highly coincident results verify that these defect modes could be used to identify defect forms caused by different factors successfully.

\section{Case study and result}

A case study with real parts is necessary to evaluate and verify the geometrical deviation identification and prediction method for AM with the defect modes proposed. This study designed a test part composed by three cylinders with height $20 \mathrm{~mm}$ and radiuses from $10-30 \mathrm{~mm}$, respectively, as Figure 22 shows to study the geometrical deviation with different design parameters.

The Makerbot Replicator 2X FDM printer, a serial machine, was applied to manufacture this model. The 1.75-mm diameter PLA filament was used for printing one part and ABS filament was used for printing two parts. Two coordinate measuring machine (CMM) machines with different measurement method were applied to better evaluate the method and modes introduced in this study: one optical coordinate measurement 
Figure 19 Possible relationship between defect modes and AM parameters

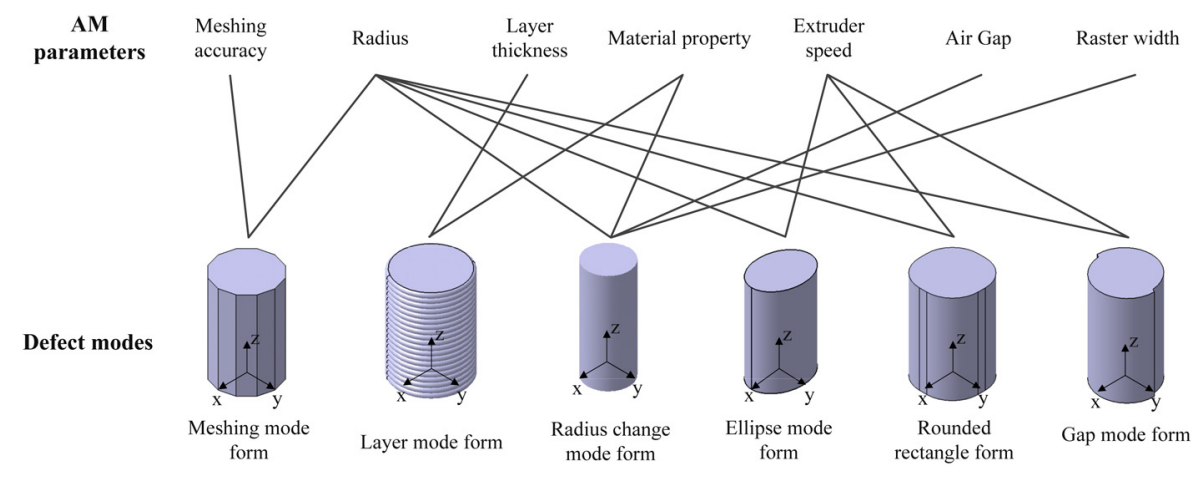

Figure 20 Defected STL files

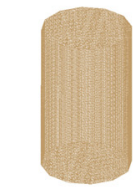

Meshing Mode defected STL file

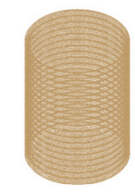

Layer Mode defected STL file

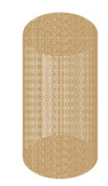

Radius Change Mode defected STL file

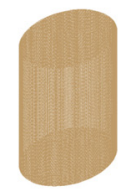

Ellipse Mode Rounded Rectangle Mode Gap mode defected STL file defected STL file defected STL file
Figure 21 Defect mode verification

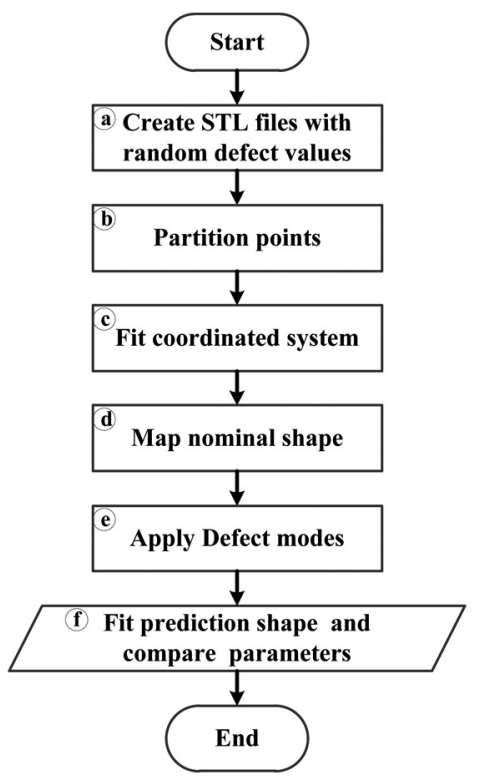

machine from GOM with accuracy less than $10 \mu \mathrm{m}$ and one contact coordinate measurement machine Renishaw SP25 with accuracy less than $4 \mu \mathrm{m}$. For each cylinder, the measurement result from GOM machine has around 100,000 points, and the measurement result from Renishaw has around 5,000 points.

Geometrical deviation identification and prediction of the test parts are then carried out with the method and modes proposed, as well as the two measurement results. The distance $(\mathrm{mm})$ between measured points and prediction points of the PLA part after applying the geometrical defect modes, as well as the deviation ( $\mathrm{mm}$ ) caused by each defect mode, is shown in Table III.

As the table shows, the differences in max distance between measured points and nominal cylinders are because CMM machine from Renishaw only measured several sections of test parts. This also causes the differences of max distance after applying the geometrical defect modes propose.

The distance between measured points and prediction points after applying the defect modes is averagely less than $0.02 \mathrm{~mm}$. The calculation results of the deviation caused by each mode with two different CMM measurements are also very close, especially for the modes describing the geometrical deviation along XY plane. This result shows that this method and defects modes could provide a good prediction of cylindrical surface geometrical deviations and could be used for identifying the deviation caused by each mode properly.

The geometrical deviation identification and prediction result of the ABS test parts measured with GOM is shown in Table IV. It could be concluded from the tables that the test parts built by FDM have poor accuracy reaching IT 12 to IT 14 , and the radius change because of the shrinkage has the largest effect on the geometrical deviation. Machine control and movement defects (ellipse mode and rounded rectangle mode), as well as machine geometrical deviation caused defect mode (gap mode), also have great influence on the part geometrical quality.

Also, the machine errors caused defect modes have the most aleatory influence on the geometrical quality, in which, the ellipse mode and the rounded rectangle mode caused deviation have the largest deviations between two parts. This shows that the control and movement accuracy of the printer need to be improved. These results could help to indicate the direction to improve the geometrical quality of $\mathrm{AM}$ manufactured parts more purposeful in future work. 
Table II Defect modes identification and verification

\begin{tabular}{|c|c|c|c|c|c|c|c|c|}
\hline \multirow[b]{2}{*}{ Geometrical defect modes } & \multirow{2}{*}{$\begin{array}{l}\text { Meshing mode } \\
\quad n_{e}(\mathrm{~mm})\end{array}$} & \multicolumn{2}{|c|}{ Layer mode } & \multirow{2}{*}{$\begin{array}{l}\text { Radius change mode } \\
\qquad R^{\prime}(\mathrm{mm})\end{array}$} & \multirow{2}{*}{$\begin{array}{l}\text { Ellipse mode } \\
\qquad R_{s}(\mathrm{~mm})\end{array}$} & \multirow{2}{*}{$\begin{array}{l}\text { Rounded rectangle mode } \\
\qquad R_{r}(\mathrm{~mm})\end{array}$} & \multicolumn{2}{|c|}{ Gap mode } \\
\hline & & $d(\mathrm{~mm})$ & $I(\mathrm{~mm})$ & & & & $d_{g}(\mathrm{~mm})$ & $\alpha\left(^{\circ}\right)$ \\
\hline Designed Value & 12 & 4 & 6 & 25 & 30 & 35 & 4 & 0.5729 \\
\hline Identified Value & 12 & 3.9999 & 6 & 25 & 29.9999 & 35 & 3.9998 & 0.5730 \\
\hline
\end{tabular}

Figure 22 CAD model, real part and measurement result of test part

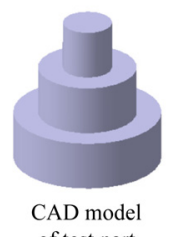

of test part

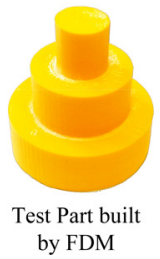

by FDM

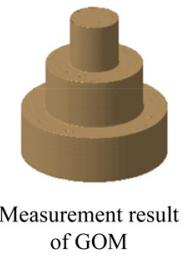

of GOM

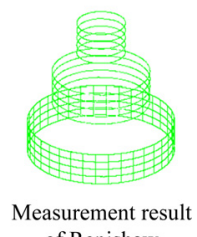
of Renishaw

\section{Conclusion}

This paper aims at analyzing the geometrical deviation of AM parts, trying to decompose the deviations based on the different defect modes appear in AM. A method to identify and predict geometrical deviations caused by different factors in $\mathrm{AM}$ is proposed to improve the geometrical quality of $\mathrm{AM}$ manufactured parts more purposefully.

This method could be used to build and extend defect modes for AM processes while identifying the geometrical defect modes caused deviations at the same time. The model error and AM processing induced errors, as well as the machine errors, have been globally taken into consideration in this work. Defect modes description based on the skin model-based modal representation has been verified to be effective with the defected STL files in this method. With the proposed modes, this method could then generate prediction shape and apply Iterative Least Square Method to identify the geometrical deviations caused by different defect modes.

In order to verify the feasibility of the method and modes proposed, case study were carried out with different radius cylinders built by FDM with two different materials. Two CMM machines with different measurement methods were used for evaluating the method and modes. The result shows that the method and modes proposed in this study could be used to identify and predict geometrical deviations caused by different defect modes successfully. The identification of geometrical deviations could also help indicate the important factors that should be optimized to improve the geometrical quality of AM.

The result of the cause study in this work has already been applied to build geometrical simulation tool for studying the impacts of the defect modes caused deviations on the geometrical behavior of the AM products. Also, this work would be applied as the base to realize the improvement of AM part geometrical quality in design phase. We have already finished the design of experiments, based on which, we are now carrying out the identification of the relationship between the defect modes parameters and process parameters, as well as input model parameters. In future work, this work would be applied to identify the deviation of AM parts with complex geometries, such as curvatures, sharpened edges and hollowed elements. Based on this work, prediction model could be proposed, and the AM part input model correction method could be carried out more

Table III Prediction and defect modes caused deviations of PLA part with two measurements (mm)

\begin{tabular}{|c|c|c|c|c|c|c|}
\hline \multirow[b]{2}{*}{ Measurements with different CMM machines } & \multicolumn{2}{|c|}{ Radius 10} & \multicolumn{2}{|c|}{ Radius 20} & \multicolumn{2}{|c|}{ Radius 30} \\
\hline & GOM & Renishaw & GOM & Renishaw & GOM & Renishaw \\
\hline \multicolumn{7}{|c|}{ Distance between measured points and nominal cylinders } \\
\hline Max distance & 0.254 & 0.183 & 0.279 & 0.244 & 0.366 & 0.306 \\
\hline Average distance & 0.106 & 0.107 & 0.155 & 0.156 & 0.233 & 0.232 \\
\hline Standard deviation & 0.053 & 0.053 & 0.043 & 0.042 & 0.051 & 0.049 \\
\hline IT class & IT13 & IT12 & IT13 & IT12 & IT13 & IT13 \\
\hline \multicolumn{7}{|c|}{ Distance between measured points and prediction points after applying the geometrical defect modes } \\
\hline Max distance & 0.168 & 0.072 & 0.148 & 0.088 & 0.130 & 0.075 \\
\hline Average distance & 0.019 & 0.018 & 0.017 & 0.015 & 0.021 & 0.019 \\
\hline Standard deviation & 0.016 & 0.013 & 0.014 & 0.011 & 0.018 & 0.014 \\
\hline \multicolumn{7}{|l|}{ Deviation caused by each geometrical defect mode } \\
\hline Mesh mode & 0.006 & 0.007 & 0.013 & 0.013 & 0.002 & 0.003 \\
\hline Layer mode & 0.002 & 0.019 & 0.002 & 0.006 & 0.005 & 0.005 \\
\hline Radius mode & 0.220 & 0.208 & 0.250 & 0.273 & 0.333 & 0.358 \\
\hline Ellipse mode & 0.105 & 0.116 & 0.074 & 0.081 & 0.072 & 0.073 \\
\hline Rounded rectangle mode & 0.028 & 0.025 & 0.035 & 0.035 & 0.043 & 0.044 \\
\hline Gap mode & 0.049 & 0.051 & 0.052 & 0.049 & 0.040 & 0.042 \\
\hline
\end{tabular}


Table IV Prediction and defect modes caused deviations of two ABS parts (mm)

\begin{tabular}{|c|c|c|c|c|c|c|}
\hline \multirow[b]{2}{*}{ Measurements of different parts } & \multicolumn{2}{|c|}{ Radius 10} & \multicolumn{2}{|c|}{ Radius 20} & \multicolumn{2}{|c|}{ Radius 30} \\
\hline & Part 1 & Part 2 & Part 1 & Part 2 & Part 1 & Part 2 \\
\hline \multicolumn{7}{|c|}{ Distance between measured points and nominal cylinders } \\
\hline Max distance & 0.177 & 0.252 & 0.32 & 0.353 & 0.661 & 0.573 \\
\hline Average distance & 0.079 & 0.131 & 0.172 & 0.234 & 0.338 & 0.383 \\
\hline Standard deviation & 0.043 & 0.050 & 0.058 & 0.049 & 0.080 & 0.064 \\
\hline IT class & IT12 & IT13 & IT13 & IT13 & IT14 & IT14 \\
\hline \multicolumn{7}{|c|}{ Distance between measured points and prediction points after applying the geometrical defect modes } \\
\hline Max distance & 0.094 & 0.127 & 0.141 & 0.128 & 0.179 & 0.175 \\
\hline Average distance & 0.017 & 0.022 & 0.018 & 0.019 & 0.029 & 0.019 \\
\hline Standard deviation & 0.017 & 0.020 & 0.020 & 0.022 & 0.024 & 0.014 \\
\hline \multicolumn{7}{|c|}{ Deviation caused by each geometrical defect mode } \\
\hline Mesh mode & 0.007 & 0.007 & 0.013 & 0.013 & 0.003 & 0.003 \\
\hline Layer mode & 0.003 & 0.003 & 0.003 & 0.004 & 0.003 & 0.003 \\
\hline Radius mode & 0.112 & 0.124 & 0.292 & 0.312 & 0.419 & 0.424 \\
\hline Ellipse mode & 0.081 & 0.112 & 0.078 & 0.106 & 0.072 & 0.097 \\
\hline Rounded rectangle mode & 0.037 & 0.044 & 0.04 & 0.047 & 0.044 & 0.054 \\
\hline Gap mode & 0.048 & 0.044 & 0.041 & 0.045 & 0.041 & 0.045 \\
\hline
\end{tabular}

purposively to improve the geometrical quality of AM parts in further study.

\section{References}

Agrawal, S. and Dhande, S.G. (2007), "Analysis of mechanical error in a fused deposition process using a stochastic approach", International fournal of Production Research, Vol. 45 No. 17, pp. 3991-4012, doi: 10.1080/00207540600791624.

Agrawal, S. and Dhande, S.G. (2008), "Analysis of geometrical error in the stereolithography process using a stochastic approach", International fournal of Production Research, Vol. 46 No. 13, pp. 3537-3562, doi: 10.1080/00207540601055458.

Agrawal, S., Deon, J.D.B. and Kumar Modi, Y. (2014), "Conversion of a GIS surface data directly to a 3D STL part for terrain modeling", Rapid Prototyping fournal, Vol. 20 No. 5, pp. 422-430, doi: 10.1108/RPJ-10-2012-0093.

Ballu, A. and Mathieu, L. (1993), "Analysis of dimensional and geometrical specifications: standards and models", Proceedings of 3rd CIRP Seminar on Computer Aided Tolerancing, Cachan.

Boschetto, A., Giordano, V. and Veniali, F. (2013), "3D roughness profile model in fused deposition modelling”, Rapid Prototyping fournal, Vol. 19 No. 4, pp. 240-252, doi: 10.1108/ 13552541311323254.

Campanelli, S.L., Cardano, G., Giannoccaro, R., Ludovico, A. D. and Bohez, E.L. (2007), "Statistical analysis of the stereolithographic process to improve the accuracy", Computer-Aided Design, Vol. 39 No. 1, pp. 80-86.

Dantan, J.-Y., Huang, Z., Goka, E., Homri, L., Etienne, A., Bonnet, N. and Rivette, M. (2017), "Geometrical variations management for additive manufactured product", CIRP Annals Manufacturing Technology, Vol. 66 No. 1, pp. 161-164.

Formosa, F. and Samper, S. (2007), "Modal expression of form defects”, in Davidson, J.K. (Ed.), Models for Computer Aided Tolerancing in Design and Manufacturing, Springer, Dordrecht, pp. 13-22.
Gao, W., Zhang, Y., Ramanujan, D., Ramani, K., Chen, Y., Williams, C.B., Wang, C.C.L., Shin, Y.C., Zhang, S. and Zavattieri, P.D. (2015), "The status, challenges, and future of additive manufacturing in engineering", Computer-Aided Design, Vol. 69, pp. 65-89, doi: 10.1016/j.cad.2015.04.001.

Gibson, I., Rosen, D.W. and Stucker, B. (2010a), Additive Manufacturing Technologies, Springer US, Boston, MA.

Gibson, I., Rosen, D.W. and Stucker, B. (2010b), "Development of additive manufacturing technology", Additive Manufacturing Technologies, Springer, pp. 36-58.

Gowda, R.B.S., Udayagiri, C.S., Narendra, D.D., Gowda, R. B.S., Udayagiri, C.S. and Narendra, D.D. (2014), "Studies on the process parameters of rapid prototyping technique (stereolithography) for the betterment of part quality, studies on the process parameters of rapid prototyping technique (stereolithography) for the betterment of part quality", International fournal of Manufacturing Engineering, Vol. 2014, p. e804705, doi: 10.1155/2014/804705.

Guo, N. and Leu, M.C. (2013), "Additive manufacturing: technology, applications and research needs", Frontiers of Mechanical Engineering, Vol. 8 No. 3, pp. 215-243, doi: 10.1007/s11465-013-0248-8.

Homri, L., Dantan, J.-Y. and Levasseur, G. (2016), "Comparison of optimization techniques in a tolerance analysis approach considering form defects", Procedia CIRP, 14th CIRP CAT 2016 - CIRP Conference on Computer Aided Tolerancing, Vol. 43, pp. 184-189, doi:10.1016/j. procir.2016.02.022

Homri, L., Goka, E., Levasseur, G. and Dantan, J.-Y. (2017), "Tolerance analysis - form defects modeling and simulation by modal decomposition and optimization", Computer-Aided Design, Vol. 91, pp. 46-59, doi: 10.1016/j.cad.2017.04.007.

Huang, W. and Ceglarek, D. (2002), "Mode-based decomposition of part form error by discrete-cosinetransform with implementation to assembly and stamping system with compliant parts”, CIRP Annals Manufacturing Technology, Vol. 51 No. 1, pp. 21-26. 
ISO/ASTM52900-15 (2015), "Standard terminology for additive manufacturing - general principles - terminology", ASTM International, West Conshohocken, PA, available at: www.astm.org.

Lecompte, J., Legoff, O. and Hascoet, J.-Y. (2010), "Technological form defects identification using discrete cosine transform method", The International fournal of Advanced Manufacturing Technology, Vol. 51 Nos 9/12, pp. 1033-1044.

Lieneke, T., Denzer, V., Adam, G.A.O. and Zimmer, D. (2016), "Dimensional tolerances for additive manufacturing: experimental investigation for fused deposition modeling", Procedia CIRP, Vol. 43, pp. 286-291, doi: 10.1016/j. procir.2016.02.361.

Lieneke, T., Adam, G.A.O., Leuders, S., Knoop, F., Josupeit, S., Delfs, P., Funke, N. and Zimmer, D. (2015), "Systematical determination of tolerances for additive manufacturing by measuring linear dimensions", 26 Th Annual International Solid Freeform Fabrication Symposium, Austin.

Morière, S., Mailhé, J., Linares, J.-M. and Sprauel, J.-M. (2010), "Assembly method comparison including form defect”, in Giordano, Lucthieu, X. and Villeneuve, F. (Eds), Product Lifecycle Management, John Wiley \& Sons, pp. 245-257, doi:10.1002/9781118557921.ch13.

Moroni, G., Syam, W.P. and Petrò, S. (2014), "Towards early estimation of part accuracy in additive manufacturing", Procedia CIRP, Vol. 21, pp. 300-305, doi: 10.1016/j. procir.2014.03.194.

Moylan, S., Slotwinski, J., Cooke, A., Jurrens, K. and Donmez, M.A. (2014), "An additive manufacturing test artifact", Fournal of Research of the National Institute of Standards and Technology, Vol. 119, p. 429, doi: 10.6028/jres.119.017.

Neri Volpato, J., Foggiatto, A. and Daniel Coradini, S. (2014), "The influence of support base on FDM accuracy in Z", Rapid Prototyping fournal, Vol. 20 No. 3, pp. 182-191, doi: 10.1108/RPJ-12-2012-0116.

Qiao, L., Wu, J., Zhu, Z. and Cui, Y. (2016), “Approach to the deviation representation of non-ideal cylindrical surfaces based on the curvilinear coordinate system", Procedia CIRP, Vol. 43, pp. 17-22, doi: 10.1016/j.procir.2016.02.008.

Sahu, R.K., Mahapatra, S.S. and Sood, A.K. (2013), “A study on dimensional accuracy of fused deposition modeling
(FDM) processed parts using fuzzy logic", fournal for Manufacturing Science and Production, Vol. 13 No. 3, p. 13, doi: 10.1515/jmsp-2013-0010.

Samper, S. and Formosa, F. (2007), "Form defects tolerancing by natural modes analysis", fournal of Computing and Information Science in Engineering, Vol. 7 No. 1, p. 44, doi: 10.1115/1.2424247.

Schleich, B., Anwer, N., Mathieu, L. and Wartzack, S. (2016), "Status and prospects of skin model shapes for geometric variations management”, Procedia CIRP, Vol. 43, pp. 154-159, doi: 10.1016/j.procir.2016.02.005.

Shahrain, M., Didier, T., Lim, G.K. and Qureshi, A.J. (2016), "Fast deviation simulation for "fused deposition modeling" process”, Procedia CIRP, Vol. 43, pp. 327-332, doi: 10.1016/ j.procir.2016.02.004.

Song, S., Wang, A., Huang, Q. and Tsung, F. (2014), "Shape deviation modeling for fused deposition modeling processes", 2014 IEEE International Conference on Automation Science and Engineering (CASE), IEEE, pp. 758-763.

Tong, K., Joshi, S. and Amine Lehtihet, E. (2008), "Error compensation for fused deposition modeling (FDM) machine by correcting slice files", Rapid Prototyping fournal, Vol. 14 No. 1, pp. 4-14, doi: 10.1108/13552540810841517.

Vayre, B., Vignat, F. and Villeneuve, F. (2012), "Metallic additive manufacturing: state-of-the-art review and prospects", Mechanics E Industry, Vol. 13 No. 2, pp. 89-96.

Wohlers, T. (2016), Wohlers report 2016, Wohlers Associates.

Xu, L., Huang, Q., Sabbaghi, A. and Dasgupta, T. (2013), "Shape deviation modeling for dimensional quality control in additive manufacturing”, ASME 2013 International Mechanical Engineering Congress and Exposition, American Society of Mechanical Engineers, pp. V02AT02A018V02AT02A018.

Yang, L. and Anam, M.A. (2014), "An investigation of standard test part design for additive manufacturing", Proceeding of the Solid Free Form Fabrication Symposium, August.

\section{Corresponding author}

Zhicheng Huang can be contacted at: Zhicheng.huang@, ensam.eu 